\title{
Prevalence of hypertension and its risk factors among cotton textile workers in low- and middle-income countries: a protocol for a systematic review
}

Naureen Akber Ali ${ }^{* *}$ and Anam Shahil Feroz ${ }^{2}$

\begin{abstract}
Background: Cotton workers are exposed to various hazards in the textile industry that might result in different ailments including hypertension (HTN). However, few attempts have been made to systematically review the prevalence of hypertension and its risk factor among cotton textile workers in low-and middle-income countries (LMICS). The objective of this study will be to evaluate the prevalence of hypertension and its risk factors among adult cotton textile workers in low- and middle-income countries.

Methods: We designed and registered a study protocol for a systematic review of descriptive epidemiology data. We will include observational studies (e.g., cross-sectional, cohort, surveys) on the epidemiology of hypertension among adult cotton textile workers in low- and middle-income countries. The primary outcome will be the prevalence of hypertension. Secondary outcomes will be the prevalence of risk factors of hypertension. Literature searches will be conducted in multiple electronic databases (from January 2000 onwards), including PubMed/ MEDLINE, CINAHL, Science Direct, and Cochrane Library. Gray literature will be identified through searching conference abstracts, thesis dissertations, and public repositories. Two investigators will independently screen all citations, full-text articles, and abstract data. The study methodological quality (or bias) will be appraised using an appropriate tool. If feasible, we will conduct random effects meta-analysis. Additional analyses will be conducted to explore the potential sources of heterogeneity (e.g., age, gender, years of service, textile department).
\end{abstract}

Discussion: This systematic review will identify, evaluate, and integrate evidence on the prevalence and risk factors of hypertension among adult cotton textile workers in low- and middle-income countries. Our findings will be made publicly available in a repository and published in a peer-reviewed journal.

Systematic review registration: PROSPERO CRD42020167175

Keywords: Blood pressure, Hypertension, Risk factors, Prevalence, Adult workers, Low- and middle- income countries, Systematic review, Cotton textile workers

\footnotetext{
* Correspondence: naureenalimeghani@gmail.com

'School of Nursing and Midwifery, The Aga Khan University, Stadium Road, PO Box 3500, Karachi 74800, Pakistan

Full list of author information is available at the end of the article
}

(c) The Author(s). 2020 Open Access This article is licensed under a Creative Commons Attribution 4.0 International License, which permits use, sharing, adaptation, distribution and reproduction in any medium or format, as long as you give appropriate credit to the original author(s) and the source, provide a link to the Creative Commons licence, and indicate if changes were made. The images or other third party material in this article are included in the article's Creative Commons licence, unless indicated otherwise in a credit line to the material. If material is not included in the article's Creative Commons licence and your intended use is not permitted by statutory regulation or exceeds the permitted use, you will need to obtain permission directly from the copyright holder. To view a copy of this licence, visit http://creativecommons.org/licenses/by/4.0/. The Creative Commons Public Domain Dedication waiver (http://creativecommons.org/publicdomain/zero/1.0/) applies to the data made available in this article, unless otherwise stated in a credit line to the data. 


\section{Background}

Globally, hypertension (HTN) is identified as one of the leading causes of mortality and disability [1]. It has led to 7.5 million deaths and accounted for 57 million disabilityadjusted life years (DALYs) or 3.7\% of total DALYs [2]. Hypertension is characterized as a constant rise of systolic blood pressure and/or diastolic blood pressure of more than or equivalent to $140 \mathrm{mmHg}$ and/or $90 \mathrm{mmHg}$ respectively [3]. Currently, around 1.13 billion individuals are suffering from hypertension globally and two-third of them resides in low- and middle-income countries (LMICs) [4] while LMICs' selection is based on World Bank's (WB) 2018 Country Classification list, having GNI per capita between $\$ 996$ and $\$ 3895$ [5]. The number of hypertensive people is projected to grow by 70 million individual from 2000 to 2025 in high-income countries (HIC) while it is estimated to grow by $>500$ million in LMICs over the same period [6]. A systematic review included data on 1,494,609 adults from 45 countries also showed that there is the highest burden of hypertension (32\%) in LMICs and prevalence estimates were found to be higher in elders, overweight/obese, urban settlers, and having informal education [7]. Moreover, worldwide high blood pressure is one of the major risk factors for cardiovascular diseases (CVD) that refers to diseases of the heart and blood vessels $[8,9]$, stroke, coronary artery disease, and peripheral vascular disease [9]. The burden of CVD across the world is very high and in LMICs, $47 \%$ of mortality secondary to CVDs and 44\% of CVDs' burden are attributable to HTN/high blood pressure (BP) [9, 10]. Generally, it is asymptomatic and the primary presentation can be seen with a devastating event like stroke or heart disease [11]. It is estimated that $45 \%$ of deaths with heart disease and $51 \%$ of deaths with stroke are related to hypertension [12]. This is the reason that it is referred to as the "silent killer" [11].

Worldwide, the textile industry has more than 60 million employees [13] who are involved in the production of fibers, yarns, fabrics, clothing, and textile products for domestic and decoration, along with the technical and industrial purposes [14]. Moreover, the textile industry involves a cluster of related industries that utilize a range of natural fibers (cotton, wool, silk, etc.) and/or synthetic fibers to generate fabric [15] which eventually produces varying amounts of dust [16]. Workers are exposed to various hazards in textile industry that might result in different ailments [17, 18]. Studies reveal high level of oxidative stress in cotton textile workers [19] leading to the development of essential hypertension [20, 21]. A study conducted in Kannur district in India found out that the prevalence of hypertension was $22.3 \%$ among cotton textile workers which was equivalent to the prevalence of general population [22]. Furthermore, increasing age, alcohol consumption, family history of hypertension, $\mathrm{BMI}>25 \mathrm{~kg} / \mathrm{m}^{2}$, and high waist-hip ratio were identified as a strong factor of hypertension among cotton workers [22]. Similarly, a study conducted in Dhaka, Bangladesh, notified that both general and central obesity were found to be significantly associated with hypertension [23]. Additionally, factors such as work-related stress, noise pollution, lack of earplug usage, tobacco smoking, and long duration of employment contribute towards the development of hypertension among the cotton textile workers [22, 24-27]. While stress in job also has a negative impact on worker's cardiovascular health which in turn will affect their job performance [28]. However, the association between cotton dust and increased blood pressure may not be strong, as confounding factors and bias impact could not be excluded [18, 29].

Chronic diseases make workers less productive, more prone to injury that leads to significant suffering, and add huge financial and service challenges on health care systems [23, 30-32]. Furthermore, it is witnessed that collective monetary losses related with the hypertension and diabetes mellitus (DM) in low- and middle-income countries are estimated to be over $\$ 7$ trillion during the years 2011-2025 that move millions of individuals below the poverty line [33]. Hypertension accounts for the global burden of cardiovascular disease which evidently has huge impact on individuals, organizations, and country in terms of economy [11]. Therefore, early detection of hypertension by identifying its risk factors is essential among highrisk population particularly in LMIC. Thus, the objective of this study will be to evaluate the prevalence of hypertension and its risk factors among adult cotton textile workers in low- and middle-income countries.

\section{Methods}

The present protocol has been registered within the PROSPERO database (registration ID: CRD42020167175). The present study protocol is being reported in accordance with the reporting guidance provided in the Preferred Reporting Items for Systematic Reviews and MetaAnalyses Protocols (PRISMA-P) statement [34] (see PRISMA-P checklist in Additional file 1).

\section{Eligibility criteria}

Studies will be selected according to the following criteria: participants, outcome(s) of interest, study design, and context.

\section{Participants (population)}

We will include studies involving adult cotton textile workers (regardless of gender). We will exclude adults working in other industries (e.g., oil mill, steel mill, and coal mill). 


\section{Condition or outcome(s) of interest}

The primary outcome will be the prevalence of hypertension (or high blood pressure) indicating the number of people that has the condition divided by the population number at a given point in time. This is often presented as a (prevalence) proportion. We will use authorreported definitions (according to definitions used in the included studies e.g., accepted diagnostic criteria or selfreport). Secondary outcomes will be the individual risk factor of hypertension. Potential individual risk factors may include the following: being overweight or obese, physically inactive, using tobacco, excessive alcohol intake, excessive salt (sodium) in the diet, too little potassium in the diet, stress, or certain chronic conditions (such as kidney disease, diabetes, and sleep apnea).

\section{Study design and context}

Eligible studies will be observational studies (cohort, crosssectional, or health surveys) reporting prevalence data using validated or non-validated tools and conducted in low- and middle-income countries. Cross-sectional studies will be the most appropriate study design to determine the prevalence of hypertension. Cross-sectional health surveys are typically used to estimate the point prevalence of common conditions of long duration. For cohort studies, only the first phase (cross-sectional) data will be considered. We will exclude randomized trials, quasi-experimental studies, case studies, case series, qualitative studies, systematic review, protocols, commentaries, and editorials.

\section{Context/settings}

We will include studies conducted in low- and middleincome countries. No limitations will be imposed on publication status (unpublished studies will be eligible for inclusion e.g., conference proceedings, abstracts). We will only include studies published in English, from January 2000 onwards.

\section{Information sources and search strategy}

The primary source of literature will be a structured search of major electronic databases (from January 2000 onwards): MEDLINE (PubMed), CINAHL Plus, Science Direct, and Cochrane Library. The secondary source of potentially relevant material will be a search of the gray or difficult to locate literature, including conference abstracts from selected national or international meeting, thesis dissertations, or documents in public repositories. We will perform handsearching of the reference lists of included studies, relevant reviews, or other relevant documents. The literature searches will be designed and conducted by the review team. The search terms will be grouped into the following categories of interest: population (e.g., cotton textile workers, factory workers, and cotton workers), epidemiological studies (e.g., prevalence, cross-sectional), outcomes (e.g., hypertension, blood pressure, systolic blood pressure, diastolic blood pressure, high blood pressure and HTN, risk factors, factors, predictors), and settings (e.g., low- and middle-income countries). Additionally, indexed keywords in the Medical Subject Headings (MeSH) will be used in order to ensure uniform search terms. The search strategy will be piloted to ensure sufficient specificity and sensitivity. A draft search strategy for PubMed/MEDLINE is provided in Additional file 2 .

\section{Screening and selection of studies}

All articles identified from the literature search will be uploaded into EndNote [35]. Records will be screened by the two reviewers (NA and AF) independently. A predefined screening tool will be designed, and a pilot testing will be conducted. First, titles and abstracts of articles returned from initial searches will be screened based on the eligibility criteria outlined above. Second, full texts will be examined in detail and screened for eligibility. Third, references of all considered articles will be hand-searched to identify any relevant report missed in the search strategy. Any disagreements will be resolved by discussion to meet a consensus, if necessary. We will contact the primary author of the study through email in case of any incomplete or missing information. A flow chart showing details of studies included and excluded at each stage of the study selection process will be provided

\section{Data collection process}

Data will be extracted onto a customized sheet in Excel that will be pilot tested prior to initiating the data extraction process. This sheet will be completed by the two independent reviewers (NA, AF) for the eligible studies. Data extraction tables filled by two the reviewers will be compared to confirm that all main findings are included. During the data extraction process, a third assessor (SF) will be involved if any discordant data is witnessed. A pilot data extraction table is provided in Additional file 3. Besides, existing studies on this research domain have been revised to assess items in the extraction form. The form included the primary author, year of publication, study setting, country of study, objective, study design, study population, male proportion, average age, BP measurement method, BP measurement apparatus, prevalence of hypertension, average $\mathrm{BP}$, risk factors of hypertension, co-morbidities, study limitations, included/excluded, and reason for exclusion, quality appraisal of included studies, and reviewer name.

\section{Evaluation of study quality}

The quality of the included studies will be evaluated by standardized quality assessment tool which will be conducted by the two authors (NA, AF). Mixed Methods Appraisal Tool (MMAT) will be used to evaluate the methodological quality of all non-randomized studies [36]. This tool is used to assess several aspects like 
selection of study participant, study tool, exposure period, missing data, measurement in outcomes, and selection of the reported result. Two reviewers (NA, AF) will rate each study as critical, serious, moderate, or low risk of bias via judgment of the gathered information. If there is limited information then the risk of bias will be categorized as "no information" or the reviewer will contact corresponding study authors for complete information.

\section{Synthesis of included studies}

The data from each paper (e.g., study characteristics, context, participants, outcomes, and findings) will be used to build evidence tables of an overall description of included studies. Crude prevalence estimates (number of cases/sample size) will be presented along with $95 \%$ confidence intervals. If feasible and appropriate, prevalence data from primary observational studies will be used to perform random effects meta-analyses. Since heterogeneity is expected a priori, we will estimate the pooled prevalence using the random effects model. The random effects model assumes the study prevalence estimates follow a normal distribution, considering both withinstudy and between-study variation. Forest plots will be used to visualize the extent of heterogeneity among studies. We will quantify statistical heterogeneity by estimating the variance between studies using $I^{2}$ statistic. The $I^{2}$ is the proportion of variation in prevalence estimates that is due to genuine variation in prevalence rather than sampling (random) error. $I^{2}$ ranges between 0 and $100 \%$ (with values of $0-25 \%$ and $75-100 \%$ taken to indicate low and considerable heterogeneity, respectively). We will also report Cochran $Q$ test with a $P$ value of $<0.05$ considered statistically significant. If sufficient studies are identified and data points are available, potential sources of heterogeneity will be investigated further by subgroup analyses according to baseline characteristics and methodological covariates. We plan to conduct analyses by gender (male vs. female), age (e.g., young adult, middle-age adult, vs. older adult), years of service, and textile department.

\section{Meta-biases}

If data permits, small study effects (publication bias) will be assessed by inspection of the funnel plots for asymmetry and with Egger's test and Begg's test, with the results considered to indicate potential small study effects when $P$ values $<0.10$.

\section{Discussion}

This review will highlight the proportion of hypertension along with its risk factor among cotton textile workers in LMICs. Given that, some of the modifiable factors may be prevented in this high-risk population, we will call on health experts including policy makers, stakeholders, cardiovascular epidemiologist, and occupational health and safety specialists to prioritize policies and commission and conduct screening and awareness programs to improve worker's health. Besides, this review will highlight an urgent need for further research on hypertension in cotton textile workers that fill in some of the data gaps such as the trends and determinants of hypertension. The review findings will be made publicly available. The results of the review will be disseminated through peer-reviewed publications and presenting at professional conferences either in the form of oral or poster presentations. Any amendments made to this protocol when conducting the study will be outlined and reported in the final manuscript.

\section{Supplementary information}

Supplementary information accompanies this paper at https://doi.org/10. 1186/s13643-020-01364-z.

Additional file 1:. PRISMA-P (Preferred Reporting Items for Systematic review and Meta-Analysis Protocols) 2015 checklist: recommended items to address in a systematic review protocol*

Additional file 2:. Search strategy for PubMed/MEDLINE

Additional file 3:. Data Extraction Table

\section{Abbreviations}

HTN: Hypertension; DALYs: Disability-adjusted life years; CVD: Cardiovascular disease; LMICs: Lower-middle-income countries; DM: Diabetes mellitus; PRISMA: Preferred Reporting Items for Systematic Reviews and Meta-analyses

\section{Acknowledgements}

The authors would like to thank Ms. Salima Farooq, Senior Instructor, School of Nursing and Midwifery Department, The Aga Khan University, Pakistan, for providing supervision as a third reviewer in case of any disagreement between the two reviewers.

\section{Authors' contributions}

NA has conceived and designed the study. NA has drafted the manuscript and is the guarantor of the systematic review. AF and NA have developed the search strategy and methods of the systematic review. AF and NA have extensively reviewed the manuscript and incorporated intellectual inputs. All authors have read, and approved the final version of the manuscript.

\section{Funding}

Not applicable

Availability of data and materials

Not applicable

Ethics approval and consent to participate

Not applicable

Consent for publication

Not applicable

Competing interests

The authors declare that they have no competing interests.

\section{Author details}

'School of Nursing and Midwifery, The Aga Khan University, Stadium Road, PO Box 3500, Karachi 74800, Pakistan. ${ }^{2}$ Department of Community Health Sciences, The Aga Khan University, Stadium Road, PO Box 3500, Karachi 74800, Pakistan. 
Received: 20 February 2020 Accepted: 16 April 2020

Published online: 02 May 2020

\section{References}

1. Forouzanfar $\mathrm{MH}$, et al. Global burden of hypertension and systolic blood pressure of at least 110 to 115 mm Hg, 1990-2015. Jama. 2017;317(2):165-82.

2. World Health Organization. Global Health Observatory $(\mathrm{GHO})$ data. Raised blood pressure, Situation and trends. https://www.who.int/gho/ncd/risk_ factors/blood_pressure_prevalence_text/en/.

3. Chobanian $\mathrm{AV}$, et al. The seventh report of the joint national committee on prevention, detection, evaluation, and treatment of high blood pressure: the JNC 7 report. Jama. 2003;289(19):2560-71.

4. World Health Organization (WHO, 2019). Hypertension; Key-facts. https:// www.who.int/news-room/fact-sheets/detail/hypertension.

5. Bank W World bank country and lending groups. LOWER-MIDDLE-INCOME ECONOMIES (\$1006 TO \$3955) 2018.

6. Perkovic $\mathrm{V}$, et al. The burden of blood pressure-related disease: a neglected priority for global health. Hypertension. 2007;50(6):991-7.

7. Sarki, A.M., et al., Prevalence of hypertension in low-and middle-income countries: a systematic review and meta-analysis. Medicine, 2015. 94(50).

8. Chiolero PBA. Prevalence and control of hypertension. Lancet. 2018; 392(10155):1305-6.

9. Roth GA, et al. Global, regional, and national burden of cardiovascular diseases for 10 causes, 1990 to 2015. Journal of the American College of Cardiology. 2017;70(1):1-25.

10. Horsa BA, Tadesse Y, Engidawork E. Assessment of hypertension control and factors associated with the control among hypertensive patients attending at Zewditu Memorial Hospital: a cross sectional study. BMC Res Notes. 2019; 12(1):152.

11. Ofori SN, Obosi J. Prevalence of hypertension among office workers in a multi-national company in the Niger-Delta with the 2017 American College of Cardiology/American Heart Association Blood Pressure Guidelines. Prev Med Rep. 2019;15:100899.

12. World Health Organization. A global brief on hypertension. WHO/DCO/ WHD/2013.2. Geneva: WHO 2013. Available from http://www.who.int/ cardiovascular_diseases/publications/global_brief_hypertension/en/.

13. Forstater M. Implications of the global financial and economic crisis on the textile and clothing sector. International Labour Office, Geneva: International Labour Organization; 2009.

14. Bullón J, et al. Manufacturing processes in the textile industry. Exp Syst Fabrics Prod. 2017

15. Moses JJ, Ammayappan L. Growth of textile industry and their issues on environment with reference to wool industry. Asian Dyer. 2006;3:61-7.

16. Nafees AA, et al. Pattern and predictors for respiratory illnesses and symptoms and lung function among textile workers in Karachi. Pakistan. Occup Environ Med. 2013;70(2):99-107.

17. Ali NA, et al. Dose-response of cotton dust exposure with lung function among textile workers: MultiTex Study in Karachi, Pakistan. Int J Occup Environ Med. 2018;9(3):120.

18. Zia S, Javeed S, Jan F. Pathophysiological effects of cotton dust pollution on blood pressure in textile workers. Cell. 300:6617901.

19. Suryakar A, et al. A study of oxidative stress in cotton industry workers from Solapur city. Biomedical Research. 2010;21(3):260-4.

20. Rodrigo $R$, et al. Relationship between oxidative stress and essential hypertension. Hypertens Res. 2007;30(12):1159.

21. Briones AM, Touyz RM. Oxidative stress and hypertension: current concepts. Curr Hypertens Rep. 2010;12(2):135-42.

22. Ismail IM, Binub K. Prevalence of hypertension and its associated factors among cotton textile workers of Kannur. Kerala. Menoufia Medical Journal. 2016;29(4):991

23. Bhowmik B, et al. Obesity and associated type 2 diabetes and hypertension in factory workers of Bangladesh. BMC research notes. 2015;8(1):460.

24. Rizi HAY, Dehghan $\mathrm{H}$. Effects of occupational noise exposure on changes in blood pressure of workers. ARYA Atherosclerosis. 2013:183-6.

25. Starzyński Z, et al. Incidence of arterial hypertension in the population of workers in the textile industry in Lódź. Medycyna pracy. 1985;36(2):131-8.

26. Tiwai $R$, et al. Hypertension among cotton textile workers. Indian journal of public health. 2003:47(1):34-6.

27. Sumardiyono $\mathrm{S}$, et al. The association between risk factors and blood pressure in the textile industry workers. Global Medical Health Comm. 2017 . 5(3):228-35
28. Kitronza, P.L. and P. Mairiaux, Occupational stress among textile workers in the Democratic Republic of Congo. Tropical medicine and health, 2015.

29. Koskela R, Klockars M, Järvinen E. Mortality and disability among cotton mill workers. Br J Ind Med. 1990;47(6):384-91.

30. Luckhaupt SE, et al. Prevalence of obesity among US workers and associations with occupational factors. Am J Prevent Med. 2014;46(3):237-48.

31. Safety, A. and C. Council, Overweight and obesity: implications for workplace health and safety and workers' compensation. 2008: Australian Safety and Compensation Council.

32. Di Lorenzo $L$, et al. Effect of shift work on body mass index: results of a study performed in 319 glucose-tolerant men working in a Southern Italian industry. Int J Obesity. 2003;27(11):1353.

33. Bloom DE, et al. From burden to "best buys": reducing the economic impact of non-communicable disease in low-and middle-income countries. 2011. Program on the Global Demography of Aging. .

34. Moher D, et al. Preferred reporting items for systematic review and metaanalysis protocols (PRISMA-P) 2015 statement. Syst Rev. 2015;4(1):1.

35. Xiehang, C.D.L., Evaluation and prospect of reference management software_- _ a case study of EndNote and NoteExpress. New Technology of Library and Information Service, 2009: p. Z1.

36. Sterne, J.A., et al., ROBINS-l: a tool for assessing risk of bias in nonrandomised studies of interventions. bmj, 2016. 355: p. i4919.

\section{Publisher's Note}

Springer Nature remains neutral with regard to jurisdictional claims in published maps and institutional affiliations.
Ready to submit your research? Choose BMC and benefit from:

- fast, convenient online submission

- thorough peer review by experienced researchers in your field

- rapid publication on acceptance

- support for research data, including large and complex data types

- gold Open Access which fosters wider collaboration and increased citations

- maximum visibility for your research: over $100 \mathrm{M}$ website views per year

At BMC, research is always in progress.

Learn more biomedcentral.com/submissions 